\title{
生物活性玻璃-二氧化锰复合支架的制备与表征
}

\author{
施吉翔 ${ }^{1}$, 翟 东 $^{2}$ ，朱 敏 ${ }^{1}$ ，朱钰方 ${ }^{2}$
}

(1. 上海理工大学 材料科学与工程学院, 上海 200093; 2. 中国科学院 上海硅酸盐研究所, 上海 200050)

摘 要: 骨修复支架在植入缺损处后出现的炎症与氧化应激有关, 其中过氧化氢 $\left(\mathrm{H}_{2} \mathrm{O}_{2}\right)$ 浓度过高是引起氧化应激的 主要原因之一。二氧化锰 $\left(\mathrm{MnO}_{2}\right)$ 能够通过催化分解 $\mathrm{H}_{2} \mathrm{O}_{2}$ 来消除植入物周围环境过量的 $\mathrm{H}_{2} \mathrm{O}_{2}$, 同时催化 $\mathrm{H}_{2} \mathrm{O}_{2}$ 分解 产生的氧气 $\left(\mathrm{O}_{2}\right)$ 能够缓解骨缺损处因血供不足而导致的缺氧环境, 从而有利于骨组织再生与骨缺损修复。本研究采 用简单的氧化还原法在 $3 \mathrm{D}$ 打印制备的生物活性玻璃(BG)支架表面原位沉积 $\mathrm{MnO}_{2}$ 颗粒, 得到 $\mathrm{BG}-\mathrm{MnO}_{2}$ 复合支架 $(\mathrm{BGM})$, 赋予 $\mathrm{BG}$ 支架清除 $\mathrm{H}_{2} \mathrm{O}_{2}$ 的同时提供 $\mathrm{O}_{2}$ 的能力。研究结果表明, $\mathrm{BGM}$ 支架表面沉积 $\mathrm{MnO}_{2}$ 含量随反应溶液 中高锰酸钾浓度升高而增加, 其抗压强度随 $\mathrm{MnO}_{2}$ 含量增加而增强, 但这些支架的孔隙率和降解速度基本保持不 变。更为重要的是, BGM 支架能够在 $\mathrm{H}_{2} \mathrm{O}_{2}$ 环境中持续催化分解 $\mathrm{H}_{2} \mathrm{O}_{2}$ 产生 $\mathrm{O}_{2}$, 当不同 $\mathrm{Mn}$ 含量的 BGM (BGM5 和 BMG9)支架在浓度为 $2 \mathrm{mmol} / \mathrm{L}$ 的 $\mathrm{H}_{2} \mathrm{O}_{2}$ 溶液中催化分解 $\mathrm{H}_{2} \mathrm{O}_{2}$ 产生的 $\mathrm{O}_{2}$ 能使溶液中饱和氧浓度分别达到 8.4 和 $11 \mathrm{mg} / \mathrm{L}$ 。细胞实验结果表明, BGM 支架对骨髓间充质干细胞的增殖和碱性磷酸酶活性有一定促进作用。因此, BGM 支架在骨组织修复领域具有较大的应用潜力。

关 键 词: 生物活性玻璃支架; 二氧化锰; 氧化应激; 三维打印; 骨组织工程

中图分类号: TQ174 文献标志码: A

\section{Preparation and Characterization of Bioactive Glass-Manganese Dioxide Composite Scaffolds}

\author{
SHI Jixiang ${ }^{1}$, ZHAI Dong ${ }^{2}$, ZHU Min ${ }^{1}$, ZHU Yufang ${ }^{2}$
}

(1. School of Materials Science and Engineering, University of Shanghai for Science and Technology, Shanghai 200093, China; 2. Shanghai Institute of Ceramics, Chinese Academy of Sciences, Shanghai 200050, China)

\begin{abstract}
Inflammation in bone defect after being implanted scaffold is related to oxidative stress, which is caused mainly by higher concentration of hydrogen peroxide $\left(\mathrm{H}_{2} \mathrm{O}_{2}\right)$. Manganese dioxide $\left(\mathrm{MnO}_{2}\right)$ can catalyze $\mathrm{H}_{2} \mathrm{O}_{2}$ decomposition to decrease excessive $\mathrm{H}_{2} \mathrm{O}_{2}$ in the surrounding environment of scaffolds. Furthermore, the oxygen $\left(\mathrm{O}_{2}\right)$ generated by the decomposition of $\mathrm{H}_{2} \mathrm{O}_{2}$ can alleviate the hypoxia caused by insufficient blood supply in bone defects, which is conducive to bone tissue regeneration. Here, a simple redox method was proposed to deposit $\mathrm{MnO}_{2}$ particles on the surface of $3 \mathrm{D}$ printed bioactive glass (BG) scaffolds for the preparation of $\mathrm{BG}-\mathrm{MnO}_{2}$ composite scaffolds (BGM), which endows BG- $\mathrm{MnO}_{2}$ scaffolds with the ability of $\mathrm{H}_{2} \mathrm{O}_{2}$ scavenging and $\mathrm{O}_{2}$ supplying simultaneously. The results showed that the $\mathrm{MnO}_{2}$ content deposited on the surface of BGM scaffolds was increased
\end{abstract}

收稿日期：2021-04-19; 收到修改稿日期：2021-06-25; 网络出版日期：2021-07-20

基金项目：国家自然科学基金(51872185); 上海理工大学科技发展项目(2020KJFZ014)

National Natural Science Foundation of China (51872185); Foundation of University of Shanghai for Science and Technology (2020KJFZ014)

作者简介: 施吉翔(1991-), 男, 硕士研究生. E-mail: 929789873@qq.com SHI Jixiang (1991-), male, Master candidate. E-mail: 929789873@qq.com

通信作者: 朱 敏，副教授.E-mail: mzhu@usst.edu.cn; 朱钰方，教授.E-mail: zhuyufang@mail.sic.ac.cn ZHU Ming, associate professor. E-mail: mzhu@usst.edu.cn; ZHU Yufang, professor. E-mail: zhuyufang@mail.sic.ac.cn 
with the increase of potassium permanganate concentration in the reaction solution, and the compressive strength of BGM scaffolds was increased with the increase of $\mathrm{MnO}_{2}$ content. However, porosity and degradation rate of these scaffolds with or without $\mathrm{MnO}_{2}$ remained the same. More importantly, BGM scaffolds can continuously catalyze the decomposition of $\mathrm{H}_{2} \mathrm{O}_{2}$ to produce $\mathrm{O}_{2}$ in $\mathrm{H}_{2} \mathrm{O}_{2}$ environment. When $\mathrm{BGM}$ with different Mn content scaffolds (BMG5 and BGM9) catalyzed the decomposition of $\mathrm{H}_{2} \mathrm{O}_{2}$ to produce $\mathrm{O}_{2}$ in $2 \mathrm{mmol} / \mathrm{L} \mathrm{H}_{2} \mathrm{O}_{2}$ solution, the saturated oxygen concentration in the solution could reach 8.4 and $11 \mathrm{mg} / \mathrm{L}$, respectively. In vitro cell experiments showed that BGM scaffolds could promote the proliferation and alkaline phosphatase activity of rabbit bone marrow mesenchymal stem cells. Hence, BGM scaffolds show great potential in bone regeneration.

Key words: bioactive glass scaffold; manganese dioxide; oxidative stress; 3D printing; bone tissue engineering

尽管骨组织工程支架在过去几十年已取得了巨 大的进步, 但是很多支架材料仍无法满足临床要 求。一方面, 骨修复支架在植入缺损处后出现的炎 症与氧化应激有关, 而过氧化氢 $\left(\mathrm{H}_{2} \mathrm{O}_{2}\right)$ 浓度过高是 引起氧化应激的主要原因之一。另一方面, 骨缺损 处因供血不足而导致的供氧不足, 严重影响了细胞 生理活动、新血管形成和组织的生长 ${ }^{[1-3]}$ 。而充足的 氧气对改善细胞黏附、生长和分化至关重要 ${ }^{[3]}$ 。在 缺氧条件下, 细胞会将葡萄糖转化为乳酸, 导致细 胞凋亡 ${ }^{[4]}$ 。目前, 无机过氧化物因能与水 $\left(\mathrm{H}_{2} \mathrm{O}\right)$ 反应 生成氧气 $\left(\mathrm{O}_{2}\right)$ 而作为释氧生物材料被用于氧气输送 的研究, 如过氧化钻、过碳酸钠和过氧化镁等 ${ }^{[1]}$ 。 Touri 等 ${ }^{[5]}$ 在双相磷酸钻支架外包覆不同浓度的过 氧化钙涂层, 研究发现 3\%过氧化钻涂层支架在低 氧条件下能提高骨细胞的生存和增殖能力。 Lü 等 ${ }^{[6]}$ 通过实验证明了混合 $20 \%$ 过氧化钙的角蛋白/丝素 蛋白支架能够在体外两周内稳定释放高水平氧, 同 时还具有抗菌功能。然而, 过氧化钙在与水反应生 成氧气的同时还产生了有毒的羟基自由基 $(\cdot \mathrm{OH})$ 而 引起氧化应激 ${ }^{[7]}$ 。因此, 有必要寻找既有 $\mathrm{H}_{2} \mathrm{O}_{2}$ 清除 能力又有释放氧气功能且无毒副作用的生物材料用 于骨修复材料复合。

通常, $\mathrm{H}_{2} \mathrm{O}_{2}$ 浓度过高是导致病灶部位炎症的主 要因素之一。二氧化锰 $\left(\mathrm{MnO}_{2}\right)$ 是一种优良的催化剂, 可以催化分解 $\mathrm{H}_{2} \mathrm{O}_{2}$ 产生氧气。将 $\mathrm{MnO}_{2}$ 引入炎症部 位可以清除过量 $\mathrm{H}_{2} \mathrm{O}_{2}$ 并产生 $\mathrm{O}_{2}$, 有效改善炎症环 境。目前已有研究表明, 含有 $\mathrm{MnO}_{2}$ 的纳米颗粒在 癌症治疗、骨关节炎症、动脉粥样硬化等疾病的 治疗方面展现出较大潜力 ${ }^{[8-11]}$ 。因此, 将 $\mathrm{MnO}_{2}$ 引入 骨修复支架, 不仅可以通过催化分解骨缺损处过 量 $\mathrm{H}_{2} \mathrm{O}_{2}$ 而改善炎症状况, 而且产生的氧气可以缓 解骨缺损处供氧不足, 从而提高细胞活力并促进骨 修复。

生物活性玻璃(BG)具有良好的生物活性、优异 的生物相容性、可降解性等特点 ${ }^{[12-13]}$ 。与生物惰性
材料不同, BG 植入物能与生物组织发生反应, 在材 料表面形成生物活性羟基磷灰石层, 使骨骼组织与 植入物间形成牢固的结合界面 ${ }^{[14-15]}$ 。3D 打印技术具 有精确控制结构的优势, 近年来在骨组织工程支架 制备领域得到广泛应用 ${ }^{[16-17]}$ 。尤其是直接墨水书写 打印, 作为一种成熟的 3D 打印技术, 具有加工灵 活、效率高、成本低、环境友好等优点 ${ }^{[18-19]}$ 。Wu 等 ${ }^{[20]}$ 将介孔生物活性玻璃(MBG) 和黏结剂聚乙烯 醇(PVA)通过挤出式 3D 打印构建骨组织工程支架, 具有高度可控的孔结构。 $\mathrm{Li}^{\text {等 }}{ }^{[21]}$ 通过 $3 \mathrm{D}$ 打印并烧 结得到的 $80 \mathrm{SiO}_{2}-15 \mathrm{CaO}-5 \mathrm{P}_{2} \mathrm{O}_{5}$ 生物玻璃支架达到人 骨小梁的平均抗压强度 $(2 \sim 12 \mathrm{MPa})$, 生物相容性良 好, 能促进骨细胞增殖与分化。因此, 利用 $3 \mathrm{D}$ 打印 技术制备 $\mathrm{BG}$ 支架并表面沉积 $\mathrm{MnO}_{2}$ 得到的 BG- $\mathrm{MnO}_{2}(\mathrm{BGM})$ 复合支架, 不仅具有可控的多孔结 构和良好的生物活性, 而且有望催化分解骨缺损处 的 $\mathrm{H}_{2} \mathrm{O}_{2}$ 而产生 $\mathrm{O}_{2}$, 有利于骨缺损修复。

已有研究报道在材料表面沉积 $\mathrm{MnO}_{2}$ 的方法有 水热法 ${ }^{[22-23]}$ 、共沉积法 ${ }^{[24]} 、$ 自生成法 ${ }^{[25-26]}$ 、氧化还 原法 ${ }^{[27-29]}$ 等。其中, 氧化还原法操作简单, 所使用的 还原剂种类繁多且经济、高效(如 PAH、MES、油酸 等)、且沉积可控。Chen 等 $^{[30]}$ 采用氧化还原法在 $\alpha-\mathrm{NaYbF}_{4}: \mathrm{Tm} @ \mathrm{CaF}_{2}$ 纳米颗粒溶液中添加 $\mathrm{MES}$ 缓 冲液和 $\mathrm{KMnO}_{4}$ 溶液, 在 $\alpha-\mathrm{NaYbF}_{4}: \mathrm{Tm} @ \mathrm{CaF}_{2}$ 颗粒 表面均匀生长 $\mathrm{MnO}_{2}$ 纳米片, 制备成复合纳米颗粒 并成功用于生物成像和生物检测。Wang 等 ${ }^{[31]}$ 在 $\mathrm{PtCo}$ 纳米粒子溶液中添加 MES 缓冲液, 溶液能够 制备具有高催化活性的单分散球形 $\mathrm{MnO}_{2} @ \mathrm{PtCo}$ 花 状结构, 其在正常和缺氧环境中对正常 NIH 3T3 细 胞几乎没有细胞毒性。因此, 氧化还原法有望实现 $\mathrm{MnO}_{2}$ 在 $\mathrm{BG}$ 支架表面的可控沉积而制备得到 $\mathrm{BGM}$ 复合支架。

基于上述研究进展, 本研究提出在 $3 \mathrm{D}$ 打印的 $\mathrm{BG}$ 支架表面, 采用简单的氧化还原法可控沉积 $\mathrm{MnO}_{2}$ 制 备 $\mathrm{BGM}$ 复合支架, 研究了 $\mathrm{MnO}_{2}$ 含量对 $\mathrm{BGM}$ 支架结 
构、理化性能以及催化分解 $\mathrm{H}_{2} \mathrm{O}_{2}$ 生成 $\mathrm{O}_{2}$ 的影响, 初 步探究了 BGM 支架的体外生物学性能。

\section{1 实验方法}

\section{1 实验材料}

正硅酸四乙酯(TEOS，98\%)、磷酸三乙酯(TEP, $99.8 \%)$ 、四水合硝酸钲 $\left(\mathrm{Ca}\left(\mathrm{NO}_{3}\right)_{2} \cdot 4 \mathrm{H}_{2} \mathrm{O}, 99 \%\right)$ 、浓盐 酸 $(\mathrm{HCl}, 37 \%)$ 、吗啉乙磺酸 $(\mathrm{MES})$ 、高锰酸钾 $\left(\mathrm{KMnO}_{4}\right)$ 、过氧化氢 $\left(\mathrm{H}_{2} \mathrm{O}_{2}, 30 \%\right)$ 均购于国药集团化 学试剂有限公司。聚乙烯醇(PVA, 分子量：146000 $186000,>99 \%$ )购于 Sigma-Aldrich。

\subsection{BGM 支架的制备}

采用溶胶一凝胶方法 ${ }^{[32]}$ 制备用于 $3 \mathrm{D}$ 打印 $\mathrm{BG}$ 支 架的 BG 粉体, 配制打印浆料前, 用行星球磨机球 磨 $\mathrm{BG}$ 粉体 $8 \mathrm{~h}$, 然后经 400 目 $(38 \mu \mathrm{m})$ 篮子过篮后备 用。3D 打印 BG 支架的粘结剂为 $10 \% \mathrm{PVA}$ 溶液, 即 将 PVA $(5 \mathrm{~g})$ 加入去离子水 $(45 \mathrm{~g})$ 后加热至 $95{ }^{\circ} \mathrm{C}$ 并 持续摚拌 $6 \mathrm{~h}$ 得到。 $\mathrm{BG}$ 支架制备过程如下: 首先将 $10 \% \mathrm{PVA}$ 溶液与 $\mathrm{BG}$ 粉末以 $1: 1$ 的重量比进行充分 混合, 形成均匀的糊状浆料; 接着将浆料转移到打 印料仓中并固定在 3-D Bioplotter ${ }^{\mathrm{TM}}$ (EnvisionTEC $\mathrm{GmbH}$, Germany)打印机上; 然后打印机通过导入 的模型控制打印头挤出一排排杆状纤维并组成栅格 状的 $\phi=10 \mathrm{~mm}$ 圆形二维平面, 相邻平面的纤维以 $60^{\circ}$ 夹角排列, 层层堆叠形成 $3 \mathrm{D}$ 多孔素坏支架, 其中 注射百的气体压力为 $250 \sim 350 \mathrm{kPa}$ ，打印速度为 4 $6 \mathrm{~mm} / \mathrm{s}$, 针头尺寸为 $400 \mu \mathrm{m}$; 最后干燥的素坏支架 在管式炉中升温至 $300{ }^{\circ} \mathrm{C}\left(1{ }^{\circ} \mathrm{C} / \mathrm{min}\right)$ 并保温 $2 \mathrm{~h}$, 再 将温度升高至 $1050{ }^{\circ} \mathrm{C}\left(3{ }^{\circ} \mathrm{C} / \mathrm{min}\right)$ 并保温 $3 \mathrm{~h}$ 得到 $B G$ 支架。

BGM 支架的制备: 据文献[33-34]方法用去离子水 配制 pH 6 的 MES 缓冲溶液(4-Morpholineethanesulfonic acid hydrate, $0.1 \mathrm{~mol} / \mathrm{L})$ 和 $\mathrm{KMnO}_{4}$ 溶液 $(0.01 \mathrm{~mol} / \mathrm{L})$ 。 将 $\mathrm{BG}$ 支架放入 MES 缓冲液超声 $10 \mathrm{~min}$, 支架质量 与 MES 体积比例为 $25 \mathrm{mg}: 1 \mathrm{~mL}$; 然后每 $10 \mathrm{~mL}$ MES 溶液中分别加入 $1 、 5 、 9 \mathrm{~mL}$ 的 $\mathrm{KMnO}_{4}$ 溶液, 继 续超声 $30 \mathrm{~min}$; 最后取出支架用去离子水冲洗三次, $60{ }^{\circ} \mathrm{C}$ 烘箱中干燥 $24 \mathrm{~h}$, 得到 $\mathrm{BGM}$ 支架。依据添加 的 $\mathrm{KMnO}_{4}$ 量依次命名为 BGM1、BGM5 和 BGM9 支架。

\subsection{BGM 支架的理化性能表征}

采用场发射电子扫描显微镜(Scanning electron microscope, SEM, FEI Quanta 450, America)观察 BGM 支架的表面形貌与微结构, 同时采用能谱仪
(Energy disperse spectroscopy, EDS)分析 BGM 支架 的表面元素组成及含量。将支架研磨成粉末后用 $\mathrm{X}$ 射 线粉末衍射仪(XRD, Bruker D8 Advance, America)测 定其广角 $\mathrm{X}$ 射线衍射图谱, $2 \theta$ 扫描范围为 $10^{\circ} \sim 80^{\circ}$, 扫 描速率为 $7\left(^{\circ}\right) / \mathrm{min}$ 。

以去离子水为介质，采用阿基米德原理测定支 架的孔隙率。孔隙率 $(P)$ 根据以下公式计算:

$$
P=\left(W_{\text {sat }}-W_{\text {dry }}\right) /\left(W_{\text {sat }}-W_{\text {sus }}\right) \times 100 \% \text {, }
$$

其中, $W_{\mathrm{dry}}$ 是支架的干重, $W_{\text {sus }}$ 是支架悬浮在水中的 重量, $W_{\text {sat }}$ 是支架浸没在去离子水中的重量。

通过万能材料试验机 (2.5 kN, Zwick-Roell, Germany) 以 $5 \mathrm{~mm} / \mathrm{min}$ 的位移速度测定支架的抗压 强度。每组 $\mathrm{BGM}$ 支架的平行样本为 3 个。

支架体外矿化：将支架与模拟体液(Simulated body fluid, SBF) 以 $200 \mathrm{~mL} / \mathrm{g}$ 的比例静置于离心管中, 到预定时间后轻轻取出支架，用去离子水清洗表面 后烘干备用。将支架用研钭研碎, 取少量粉末与 $\mathrm{KBr}$ 粉末混合压片, 采用傅里叶变换红外光谱仪 (FT-IR, PerkinElmer SPECTRUM 100, Germany)测 定矿化前后支架的 FT-IR 谱图, 谱图扫描范围为 $2000 \sim 450 \mathrm{~cm}^{-1}$ 。

支架体外降解性能：将支架浸泡在 $37{ }^{\circ} \mathrm{C}$ 的 Tris- $\mathrm{HCl}$ 缓冲溶液中, 经过 $1 、 2 、 3 、 5 、 7 、 14 、 21 、$ $28 \mathrm{~d}$ 后, 取出、洗涤、烘干后称量支架的剩余重量, 其 中 Tris- $\mathrm{HCl}$ 缓冲液体积与支架质量的比例为 $200 \mathrm{~mL} / \mathrm{g}$, 并且在每次称重后重新加入相应比例的 Tris- $\mathrm{HCl}$ 缓 冲液。另外, 将支架置于 $37{ }^{\circ} \mathrm{C}$ 的新鲜 SBF 中测试 支架所在溶液持续 $14 \mathrm{~d}$ 的 $\mathrm{pH}$ 变化, 其中 SBF 体积 与支架质量的比例为 $200 \mathrm{~mL} / \mathrm{g}$ 。

\subsection{BGM 支架催化过氧化氢产生氧气}

通过便携式溶解氧测定仪(JPB-607A)测试溶液 的饱和氧浓度。为了测定 $\mathrm{BGM}$ 支架对 $\mathrm{H}_{2} \mathrm{O}_{2}$ 的催化 产氧能力, 首先将测试氧电极探针插入配制的 $\mathrm{H}_{2} \mathrm{O}_{2}$ 溶液中以实时记录溶液的氧浓度。待数值稳定后, 将支架放入待测浓度的 $\mathrm{H}_{2} \mathrm{O}_{2}$ 溶液中，通过测定仪 上的数值实时记录溶解氧浓度随时间变化的情况。 分别测试 $\mathrm{BGM}$ 支架在不同浓度 $\mathrm{H}_{2} \mathrm{O}_{2}$ 溶液中的饱和 氧浓度变化。每组测试进行三组平行试验，其中 $\mathrm{H}_{2} \mathrm{O}_{2}$ 溶液与支架的比例为 $200 \mathrm{~mL} / \mathrm{g}$ 。

\section{5 细胞实验}

采用兔源骨髓间充质干细胞(rBMSCs)进行体 外细胞实验。细胞采用 DMEM 培养基培养, 其中 DMEM 培养基含有 10\%牛胎血清(FBS), $4.5 \mathrm{~g} / \mathrm{L}$ 的 葡萄糖 (Gibco，Carlsbad，CA) 和抗生素 (青霉素, $100 \mathrm{U} / \mathrm{mL}$; 链霉素, $0.1 \mathrm{mg} / \mathrm{mL})$ 。将灭菌锅灭菌 
$\left(121{ }^{\circ} \mathrm{C}, 30 \mathrm{~min}\right)$ 的 $\mathrm{BG}$ 和 $\mathrm{BGM}$ 支架置于 24 孔板内, 然后向每个支架滴加 $200 \mu \mathrm{L}$ 含 $1 \times 10^{5} \mathrm{rBMSCs}$ 的细胞 悬液, $30 \mathrm{~min}$ 后加入培养基没过支架, 并在 $37{ }^{\circ} \mathrm{C}$ 、 $5 \% \mathrm{CO}_{2}$ 的气氛培养箱内孵育。

通过 CCK-8(Cell counting Kit-8) 方法评估 $\mathrm{rBMSCs}$ 在支架上的增殖情况。各组支架接种 rBMSCs 后分别培养 $1 、 3$ 和 $7 \mathrm{~d}$, 向各孔中加入 $360 \mu \mathrm{L}$ 的培养 基和 $40 \mu \mathrm{L}$ 的 CCK-8 溶液，继续在 $37{ }^{\circ} \mathrm{C} 、 5 \% \mathrm{CO}_{2}$ 气氛培养箱中培养 $4 \mathrm{~h}$ 。然后在每个孔中取出 $100 \mu \mathrm{L}$ 溶液转移到新的 96 孔板中, 并使用酶标仪(BioRad 680 , USA)测试 $450 \mathrm{~nm}$ 处的 OD 值, 用以定量表征 细胞增殖情况。

通过测试碱性磷酸酶活性(Alkaline Phosphatase, ALP)的表达来分析各组支架上的细胞初期成骨分化 状况。将接种 rBMSCs 的支架培养 3 和 $7 \mathrm{~d}$ 后, 使用 $\mathrm{PBS}$ 和 Tris- $\mathrm{HCl}$ 缓冲液清洗三次。接着, 使用 $200 \mu \mathrm{L}$ 的 $0.2 \%$ 聚乙二醇辛基苯基醚(Triton X-100)溶液溶 解细胞, 超声分散后在 $4{ }^{\circ} \mathrm{C}$ 下 $14000 \mathrm{r} / \mathrm{min}$ 离心。 然后, 取 $50 \mu \mathrm{L}$ 上层清液与 $150 \mu \mathrm{L}$ 标准试剂混合, 在酶标仪 $405 \mathrm{~nm}$ 处读取 OD 值。同时, 按检测试剂 盒说明, 作蛋白定量检测, 最后碱性磷酸酶活性以 OD (min'(mg protein) $)^{-1}$ )表示。

\section{2 结果与讨论}

\subsection{BGM 支架结构表征}

图 1(A)为 $B G$ 和 $B G M$ 支架的光学照片。由图 可见, $\mathrm{BG}$ 支架为白色, 而随着制备过程中 $\mathrm{KMnO}_{4}$ 的添加量增加, BGM 支架表面颜色呈现由浅黄色到 深棕色的变化, 且表面颜色较为均匀。这说明通过 改变制备过程中 $\mathrm{KMnO}_{4}$ 的加入量, 可以调控 $\mathrm{BGM}$ 支架表面沉积 $\mathrm{MnO}_{2}$ 的量。图 1(B)为 BG 和 BGM 支 架的广角 XRD 图谱。根据图谱可知, 经烧结的 BG 支架只在 $2 \theta=20^{\circ} \sim 30^{\circ}$ 存在宽化的馒头峰, 并且没有
其他明显的衍射峰。而 BGM1、BGM5、BGM9 支 架在 $2 \theta=18.06^{\circ}$ 处存在衍射峰, 对应 $\mathrm{MnO}_{2}$ 的特征峰 (PDF-\#72-1982)。另外, 随着支架表面 $\mathrm{MnO}_{2}$ 含量的 增加, $2 \theta=18.06^{\circ}$ 处衍射峰变得更为明显, 而 $2 \theta=$ $20^{\circ} \sim 30^{\circ}$ 处的馒头峰有所下降。由此表明 BG 支架表 面成功沉积了不同含量的 $\mathrm{MnO}_{2}$ 。

图 2 为 $\mathrm{BG}$ 和 $\mathrm{BGM}$ 支架的 SEM 照片及相应的 表面 $\mathrm{EDS}$ 谱图。由图可以看出, $\mathrm{BG}$ 支架在沉积 $\mathrm{MnO}_{2}$ 前后的宏观结构没有变化, 都呈现有序排列 且三维连通的多孔结构, 表明氧化还原法沉积 $\mathrm{MnO}_{2}$ 不会破坏支架的宏观结构。另一方面, 随着沉 积过程中 $\mathrm{KMnO}_{4}$ 的添加量增加, 支架表面沉积的 细小 $\mathrm{MnO}_{2}$ 颗粒从无到有, 且逐渐增加。由 EDS 能 谱分析可知, $\mathrm{BG}$ 支架表面没有 $\mathrm{Mn}$ 元素, 而 BGM 支 架表面则探测到明显的 Mn 元素; BGM1、BGM5 和 BGM9 支架表面的 Mn 元素在支架表面的重量占比 分别为 $0.41 \% 、 0.94 \%$ 和 $1.18 \%$ 。以上结果也表明通 过氧化还原法可成功将 $\mathrm{MnO}_{2}$ 沉积在 $\mathrm{BG}$ 支架表面, 并且可以通过沉积过程中 $\mathrm{KMnO}_{4}$ 含量控制而调控 $\mathrm{MnO}_{2}$ 的沉积量。

\subsection{BGM 支架的孔隙率和机械强度}

通过阿基米德方法测试计算得到的 BG 和 BGM 支架的孔隙率如图 3(A)所示。BG、BGM1、BGM5 和 BGM9 支架的孔隙率分别为 $(78.28 \pm 0.38) \%$ 、 $(76.81 \pm 1.15) \%$ 、(78.12 \pm 0.67$) \%$ 和 $(77.53 \pm 0.97) \%$ 。各 组支架之间的孔隙率没有明显的差异, 说明 $\mathrm{BG}$ 支 架表面沉积的 $\mathrm{MnO}_{2}$ 颗粒没有对支架的连通性和大 孔结构造成影响。

$\mathrm{BG}$ 和 BGM 支架的抗压强度如图 3(B) 所示。可 以看出, 随着支架表面沉积 $\mathrm{MnO}_{2}$ 含量的增加, 支架 的抗压强度呈上升趋势。BG、BGM1、BGM5 和 BGM9 支架的抗压强度分别为 $(2.21 \pm 0.15) 、(2.61 \pm 0.32)$ 、 $(3.09 \pm 0.13)$ 和 $(3.45 \pm 0.39) \mathrm{MPa}$ 。从力学强度考虑, $\mathrm{BGM}$ 支架均在 $2 \mathrm{MPa}$ 以上, 满足人体松质骨的最
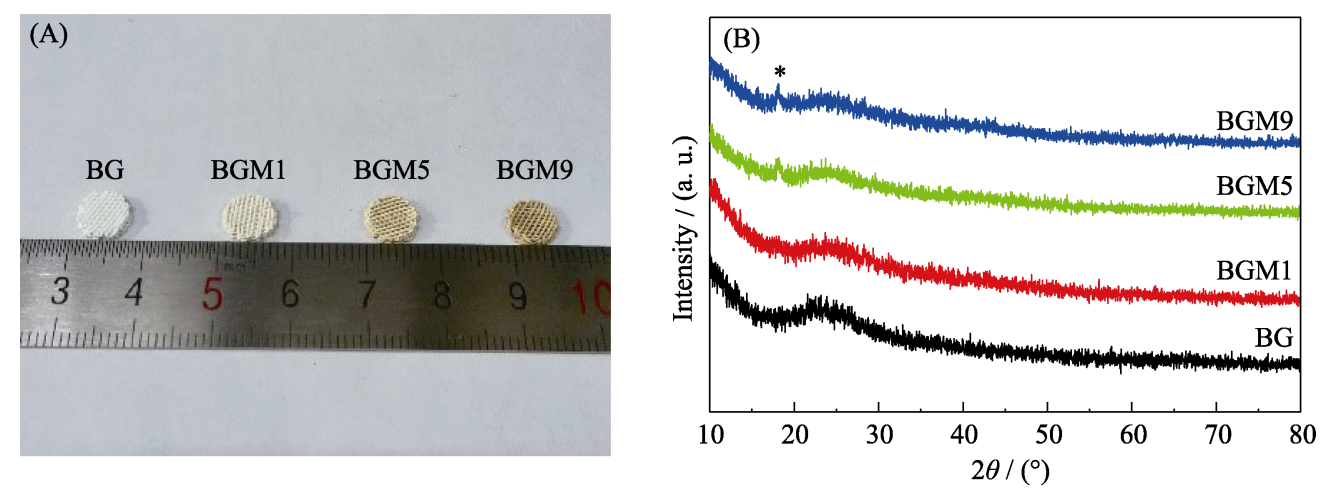

图 1 (A)BG 和 $\mathrm{BGM}$ 支架的光学照片及(B)XRD 图谱

Fig. 1 (A) Optical picture and (B) XRD patterns of BG and BGM scaffolds 

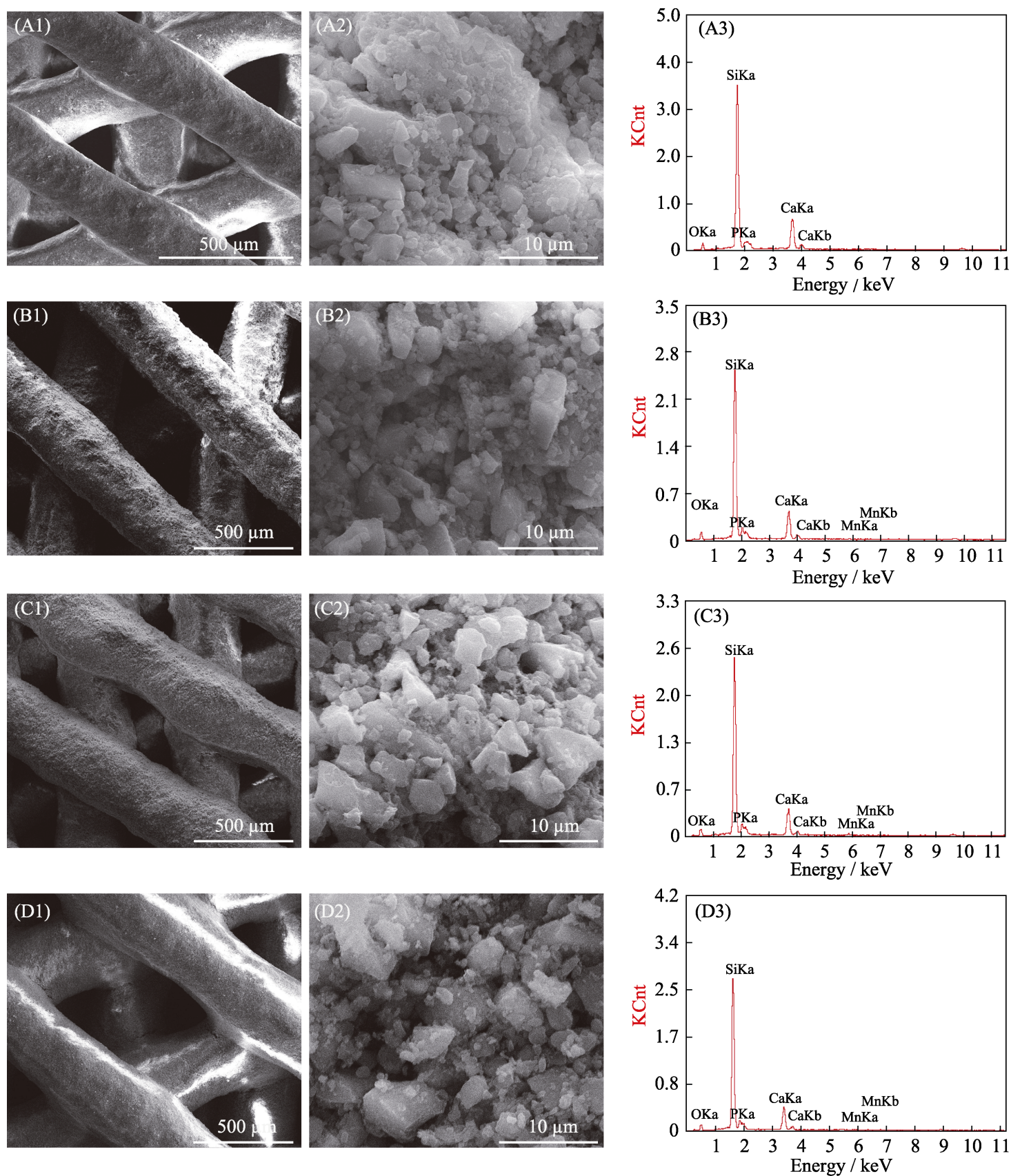

图 2 BG 和 BGM 支架的(A1, A2, B1, B2, C1, C2, D1, D2) SEM 照片和相应的(A3, B3, C3, D3)EDS 图谱

Fig. 2 (A1, A2, B1, B2, C1, C2, D1, D2) SEM images and corresponding (A3, B3, C3, D3) EDS spectra of BG and BGM scaffolds (A1, A2, A3) BG scaffold; (B1, B2, B3) BGM1 scaffold; (C1, C2, C3) BGM5 scaffold; (D1, D2, D3) BGM9 scaffold
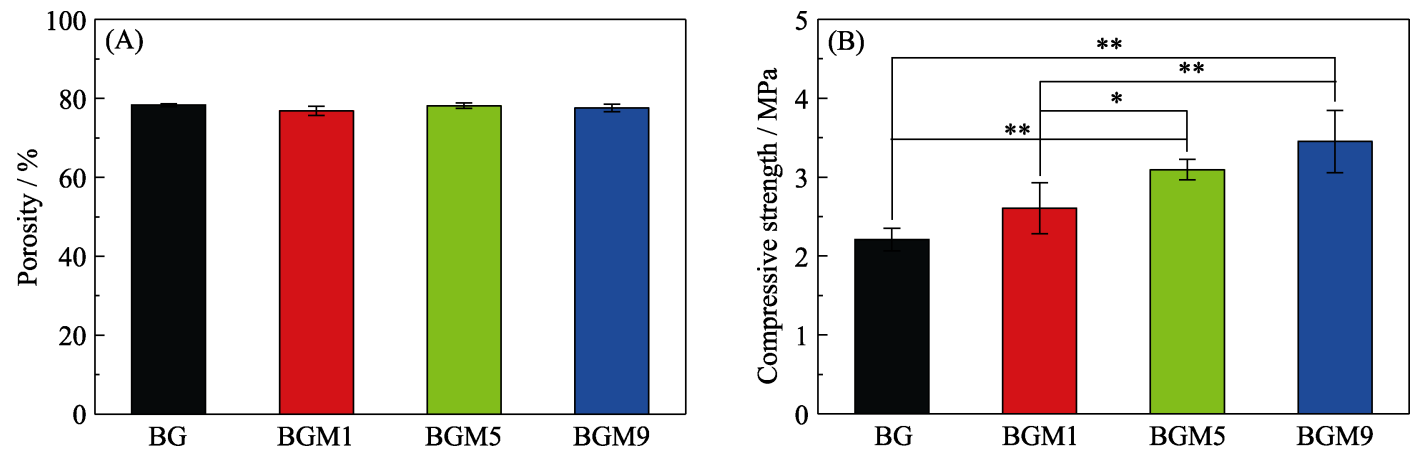

图 3 BG 和 $\mathrm{BGM}$ 支架的(A)孔隙率和(B)抗压强度

Fig. 3 (A) Porosities and (B) compressive strengths of BG and BGM scaffolds $\left(* p<0.05,{ }^{* *} p<0.01\right)$ 
低抗压强度要求 ${ }^{[35]}$ 。这里 $\mathrm{BGM}$ 支架可能因 $\mathrm{MnO}_{2}$ 细小颗粒能够沉积在支架表面的细小孔隙中而改善 支架的抗压强度。Azizi 等 ${ }^{[36]}$ 研究发现沉积 $\mathrm{MnO}_{2}$ 的羟基磷灰石复合支架也同样获得了比纯羟基磷灰 石支架更高的抗压强度

\section{$2.3 \mathrm{BGM}$ 支架的矿化、降解及 $\mathrm{pH}$ 变化}

图 4(A) 是 BG 和 BGM 支架浸泡 SBF 前的 FT-IR 谱图。由图可知, 在 $1104 、 801$ 和 $472 \mathrm{~cm}^{-1}$ 处出现 $\mathrm{Si}-\mathrm{O}$ 官能团引起的振动峰, 并且在 960、604 和 $575 \mathrm{~cm}^{-1}$ 处出现了典型 $\mathrm{PO}_{4}{ }^{3-}$ 官能团引起的特征振动峰 ${ }^{[21]}$ 。 图 4(B) 是 $\mathrm{BG}$ 和 $\mathrm{BGM}$ 支架在 $\mathrm{SBF}$ 中浸泡 $5 \mathrm{~d}$ 后的 FT-IR 谱图。相较于浸泡 SBF 前的 BG 和 BGM 支 架, 各组支架在 SBF 浸泡 $5 \mathrm{~d}$ 后的特征振动峰均有 所增强, 而且其振动峰的增强随支架表面 $\mathrm{MnO}_{2}$ 含 量的增加而更加显著, 说明 $\mathrm{MnO}_{2}$ 沉积有利于支架 表面的磷酸盐物质生成。这可能是因为支架表面沉 积的 $\mathrm{MnO}_{2}$ 颗粒可以成为羟基磷灰石成核位点, 有 利于羟基磷灰石生成。因此, BGM 支架具有生物活性。

图 5(A) 是 BG 和 BGM 支架在 Tris- $\mathrm{HCl}$ 溶液中 浸泡 $28 \mathrm{~d}$ 的降解曲线。由图可知, 各组支架的降解 速率接近, 经过 Tris- $\mathrm{HCl}$ 溶液浸泡 $28 \mathrm{~d}$, 各组支架
质量都约减 $15 \%$ 。这说明 $\mathrm{BGM}$ 支架表面的 $\mathrm{MnO}_{2}$ 对支架降解的影响较小。图 5(B)为 BG 和 BGM 支 架在 SBF 中浸泡 $14 \mathrm{~d}$ 的 $\mathrm{pH}$ 变化曲线。由图可见, 前 三天各组支架浸泡后的溶液 $\mathrm{pH}$ 呈上升趋势, 之后 其 $\mathrm{pH}$ 变化不大。这可能是由于前期支架中的 $\mathrm{Ca}$ 、 $\mathrm{P} 、 \mathrm{Si}$ 离子释放使溶液 $\mathrm{pH}$ 有一定程度上升, 而之后 支架表面沉积羟基磷灰石使得溶液中离子交换变缓, $\mathrm{pH}$ 变得相对稳定。另一方面, 浸泡 BG、BGM1、 BGM5 和 BGM9 支架的 SBF 在第三天的 $\mathrm{pH}$ 分别为 (7.54 \pm 0.01$) 、(7.53 \pm 0.02) 、(7.55 \pm 0.02)$ 和 $(7.56 \pm 0.02)$, 说明 $\mathrm{MnO}_{2}$ 作为一种两性氧化物对支架在 $\mathrm{SBF}$ 中的 $\mathrm{pH}$ 影响不大。当支架周围环境的 $\mathrm{pH}$ 与人体体液接 近或呈弱碱性时, 有利于新生骨生长, 并且稳定的 $\mathrm{pH}$ 环境也是成骨细胞黏附增殖的关键。

\section{$2.4 \mathrm{BGM}$ 支架催化 $\mathrm{H}_{2} \mathrm{O}_{2}$ 产生氧气}

$\mathrm{MnO}_{2}$ 通过催化分解 $\mathrm{H}_{2} \mathrm{O}_{2}$ 可以在溶液中产生一 定浓度的饱和氧。图 6(A) 是 BGM9 支架在不同浓度 $\mathrm{H}_{2} \mathrm{O}_{2}$ 溶液中产生的饱和氧浓度曲线, 从图中可以看 出, 随着 $\mathrm{H}_{2} \mathrm{O}_{2}$ 浓度增加, 溶液中的饱和氧浓度逐渐 升高, 在 $20 \mathrm{mmol} / \mathrm{L}$ 的 $\mathrm{H}_{2} \mathrm{O}_{2}$ 溶液中, BGM9 支架使 其饱和氧浓度最高时达到平均 $25.37 \mathrm{mg} / \mathrm{L}$, 远
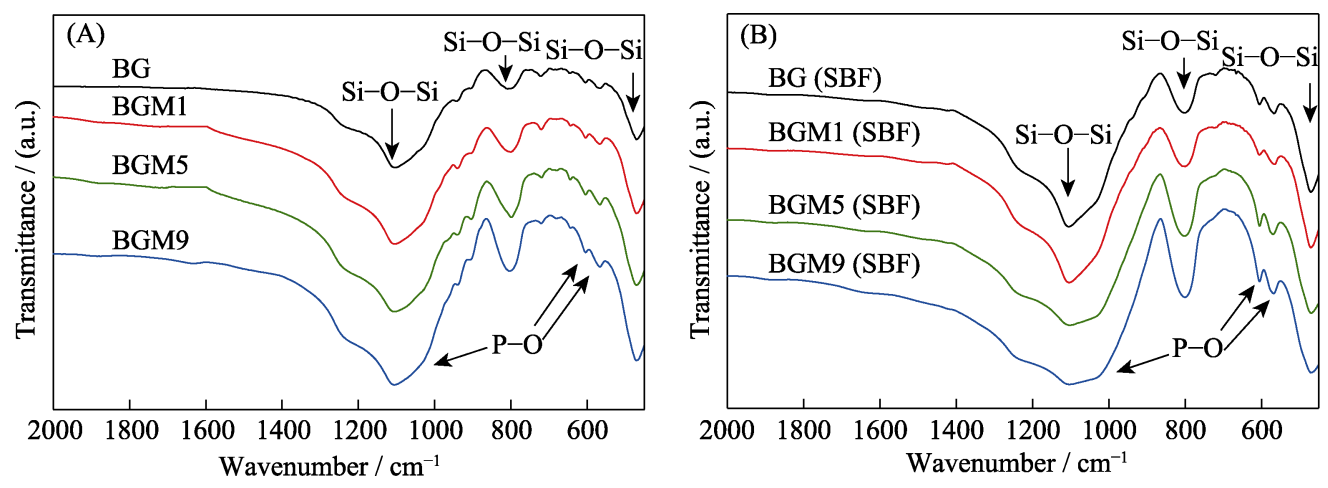

图 4 BG 和 $\mathrm{BGM}$ 支架(A)浸泡 $\mathrm{SBF}$ 前和(B)浸泡 SBF 5 d 后的 FT-IR 谱图

Fig. 4 FT-IR spectra of BG and BGM scaffolds (A) before and (B) after soaking in SBF for $5 \mathrm{~d}$
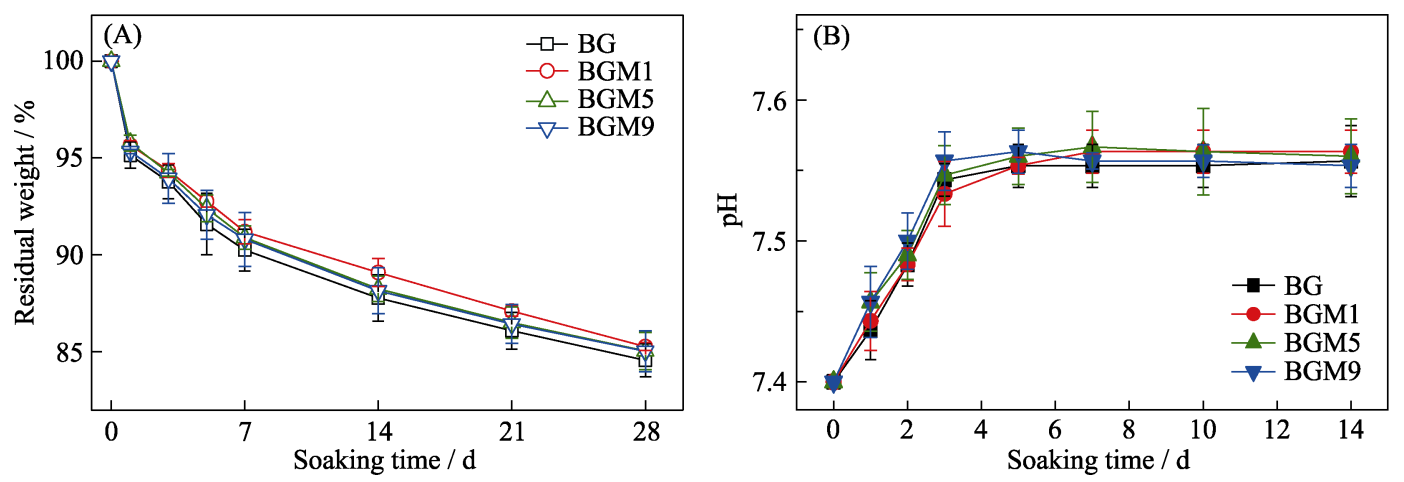

图 5 (A)BG 和 $\mathrm{BGM}$ 支架在 Tris- $\mathrm{HCl}$ 中浸泡 $28 \mathrm{~d}$ 的降解曲线及(B)BG 和 $\mathrm{BGM}$ 支架在 $\mathrm{SBF}$ 中浸泡 $14 \mathrm{~d}$ 的 $\mathrm{pH}$ 变化曲线

Fig. 5 (A) Degradation curves of BG and BGM scaffolds in Tris- $\mathrm{HCl}$ for $28 \mathrm{~d}$, and (B) $\mathrm{pH}$ change curves of SBF after BG and BGM scaffolds soaking for $14 \mathrm{~d}$ 
远高于不含 $\mathrm{H}_{2} \mathrm{O}_{2}$ 的去离子水溶液中所测得的饱和氧 浓度 $6.53 \mathrm{mg} / \mathrm{L}$ 。Hsieh 等 ${ }^{[37]}$ 研究发现 PLGA $/ \mathrm{CaO}_{2} / \mathrm{MnO}_{2}$ 复合纳米颗粒能够使溶液中的饱和氧浓度达到 10 12 mg/L，并且有效缓解低氧张力下细胞生长的 缺氧状态，促进其成骨分化。一般情况下人体内的 $\mathrm{H}_{2} \mathrm{O}_{2}$ 浓度在 1 8 $\mu \mathrm{mol} / \mathrm{L}$, 而巨噬细胞活化后可产生局 部高浓度的 $\mathrm{H}_{2} \mathrm{O}_{2}(0.01 \sim 1 \mathrm{mmol} / \mathrm{L})^{[38-39]}$ 。此外, 在炎症 性疾病中的 $\mathrm{H}_{2} \mathrm{O}_{2}$ 浓度可能达到 $20 \mathrm{mmol} / \mathrm{L}$, 这取决于 炎症环境中的中性粒细胞 ${ }^{[40]}$ 。许多 $\mathrm{H}_{2} \mathrm{O}_{2}$ 响应材料 在 $\mathrm{H}_{2} \mathrm{O}_{2}$ 浓度为 $0.02 \sim 5 \mathrm{mmol} / \mathrm{L}$ 的模拟炎症环境中进 行相关测试 ${ }^{[41-43]}$ 。本研究中 BGM9 支架能够使含 $\mathrm{H}_{2} \mathrm{O}_{2}$ 的模拟炎症环境 $(2 \mathrm{mmol} / \mathrm{L})$ 中的饱和氧浓度达到 $(11.00 \pm 0.36) \mathrm{mg} / \mathrm{L}$, 这有缓解细胞缺氧环境的效果, 有利于细胞的增殖与分化。本实验对 BGM 支架在 $\mathrm{H}_{2} \mathrm{O}_{2}(2 \mathrm{mmol} / \mathrm{L})$ 溶液中的重复循环催化释放氧气能 力进行了评估。图 6(B) 是 $\mathrm{BGM}$ 支架在含有 $\mathrm{H}_{2} \mathrm{O}_{2}$ $(2 \mathrm{mmol} / \mathrm{L})$ 溶液中重复循环三次的溶解氧变化曲 线。由图可知, BGM1、BGM5、BGM9 支架分别使 溶液的饱和氧浓度达到 $(6.93 \pm 0.12) 、(8.40 \pm 0.26)$ 和 $(11.00 \pm 0.20) \mathrm{mg} / \mathrm{L}$ 。除了 BGM1 支架处理的溶液中
饱和氧浓度很快下降到初始溶液的饱和氧浓度外, BGM5 和 BGM9 支架处理的溶液中饱和氧浓度分别 在 13 和 $16 \mathrm{~min}$ 左右才开始逐渐下降, 表明支架具 有持续催化释放氧气的能力。另外，将试验的支架 取出后再次放入到相同浓度的 $\mathrm{H}_{2} \mathrm{O}_{2}$ 溶液, 其处理 溶液中的饱和氧浓度只有略微下降, 说明 BGM 支 架表面的 $\mathrm{MnO}_{2}$ 与支架产生较强的结合力, 并且表 面沉积的 $\mathrm{MnO}_{2}$ 没有在催化过程中被产生的 $\mathrm{O}_{2}$ 破 坏。因此, BGM 支架在模拟人体炎症环境中具有持 续的催化分解 $\mathrm{H}_{2} \mathrm{O}_{2}$ 产生氧气的能力。

\subsection{BGM 支架的细胞实验}

图 7(A) 是 $\mathrm{CCK}-8$ 法检测 $\mathrm{rBMSCs}$ 在 $\mathrm{BG}$ 和 $\mathrm{BGM}$ 支架上培养 $1 、 3$ 和 $7 \mathrm{~d}$ 的细胞增殖状况，可以看到, 随着细胞培养时间延长, $\mathrm{BG}$ 和 $\mathrm{BGM}$ 支架上的细胞 明显增殖。另一方面，当细胞在各组支架上培养 3 和 $7 \mathrm{~d}$ 后, BGM1 和 BGM5 支架上细胞增长率显著高 于 $B G$ 支架，而 BGM9 支架组则显示出与 $B G$ 支架 组相近的细胞增长率, 表明 BGM1 和 BGM5 支架能 够促进 rBMSCs 增殖。细胞在 BGM 支架上的增殖 一方面取决于支架释放的具有生物活性的 $\mathrm{Si} 、 \mathrm{Ca}$
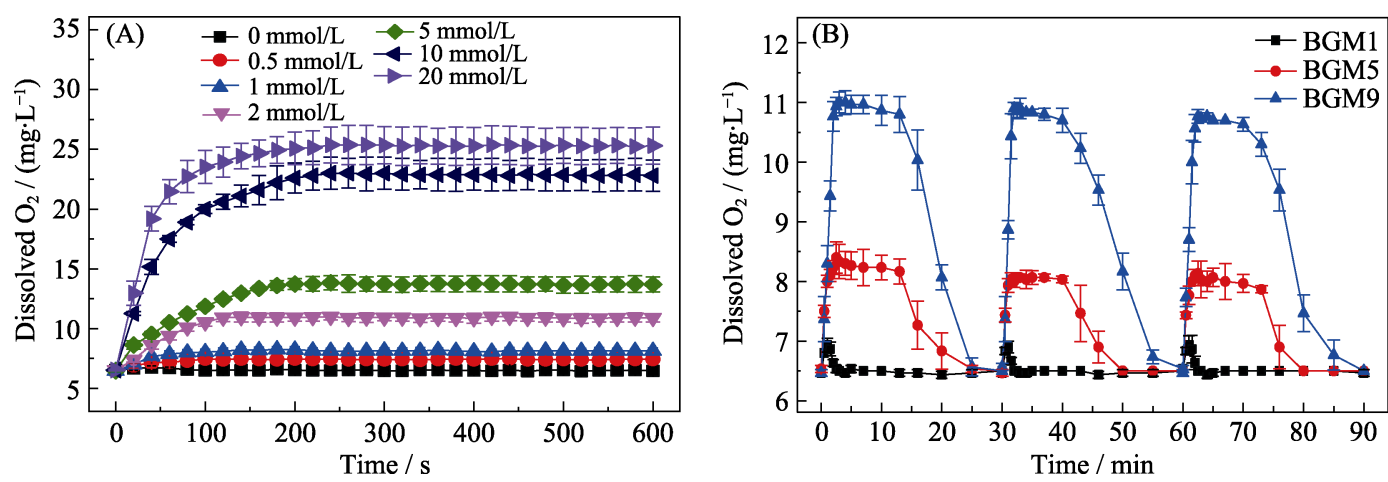

图 6 (A)BGM9 支架在不同浓度 $\mathrm{H}_{2} \mathrm{O}_{2}$ 溶液中的溶解氧水平和(B)BGM 支架在 $2 \mathrm{mmol} / \mathrm{L} \mathrm{H}_{2} \mathrm{O}_{2}$ 溶液中溶解氧变化曲线(连续循环测试 3 次)

Fig. 6 (A) Dissolved oxygen levels in different concentrations of $\mathrm{H}_{2} \mathrm{O}_{2}$ solution after immersing BGM9 scaffolds, and (B) dissolved oxygen change curves in $2 \mathrm{mmol} / \mathrm{L} \mathrm{H}_{2} \mathrm{O}_{2}$ solutions after immersing BGM scaffolds (cycle test for 3 times)
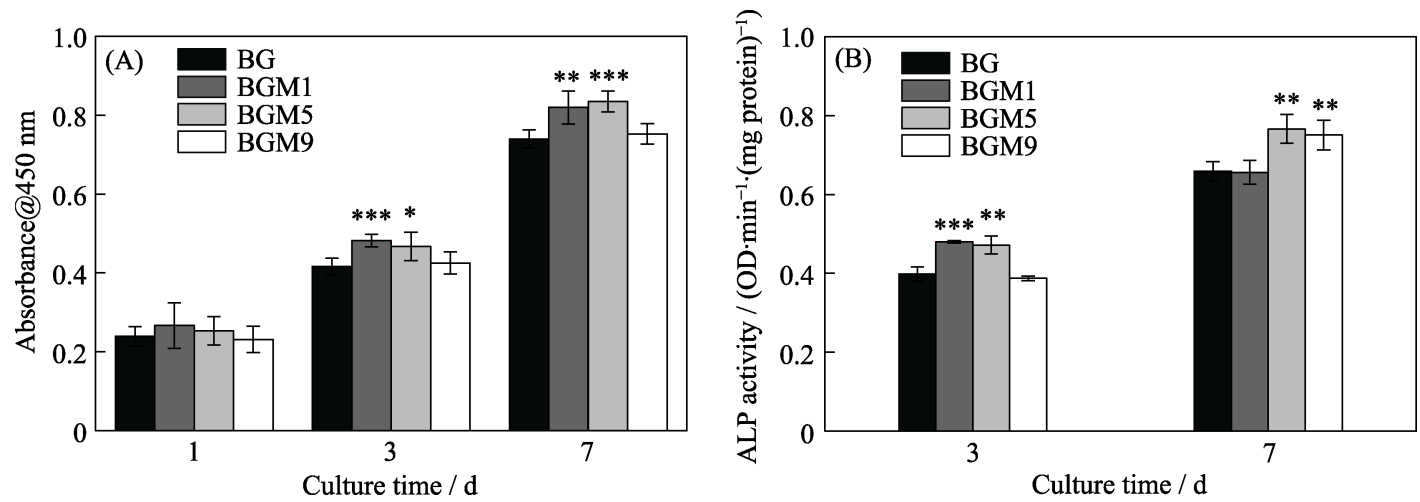

图 7 (A)rBMSCs 细胞在 BG 和 BGM 支架上的增殖和(B)ALP 活性

Fig. 7 (A) Proliferation and (B) ALP activity of rBMSCs on BG and BGM scaffolds 
离子和支架周围稳定的 $\mathrm{pH}$ 环境 ${ }^{[44-45]}$ 。另一方面, $\mathrm{BGM}$ 支架表面释放适量的 $\mathrm{Mn}$ 离子也能促进成骨细 胞的黏附与增殖 ${ }^{[46]}$ 。

通过分析细胞在 BG 和 BGM 支架上的 ALP 活 性情况, 进一步评估了 $\mathrm{rBMSCs}$ 在 BG 和 BGM 支架 上的初期成骨分化能力。图 7(B) 是 rBMSCs 在 BG 和 BGM 支架上培养 3 和 $7 \mathrm{~d}$ 的 ALP 活性表达水平。 随着细胞培养时间延长, $\mathrm{BG}$ 和 BGM 支架组的 ALP 活性都随之增大。当细胞在各组支架上培养 $3 \mathrm{~d}$ 时, BGM1 和 BGM5 支架组的 ALP 活性明显高于 BG 支架组, 而 BGM9 支架组的 ALP 活性接近 BG 支架 组。当细胞在各组支架上培养 $7 \mathrm{~d}$ 时, BGM5 和 BGM9 支架组的 ALP 活性明显高于 BG 支架组。这 说明 BGM5 支架对 rBMSCs 有较好的成骨分化促进 作用。

\section{3 结论}

本研究采用简单的氧化还原法在 3D 打印制备 的 $\mathrm{BG}$ 支架表面沉积 $\mathrm{MnO}_{2}$ 纳米颗粒, 得到了 $\mathrm{BGM}$ 复合支架。通过调节 $\mathrm{KMnO}_{4}$ 溶液浓度可以调控 BGM 支架表面 $\mathrm{MnO}_{2}$ 的沉积量。 $\mathrm{BGM}$ 支架具有规 则的连通多孔结构, 且不同 $\mathrm{MnO}_{2}$ 量的 $\mathrm{BGM}$ 支架具 有相近的孔隙率和降解速率。随着沉积 $\mathrm{MnO}_{2}$ 量的 增加, BGM 支架的抗压强度也明显增加。更为重要 的是, BGM 支架能够催化分解 $\mathrm{H}_{2} \mathrm{O}_{2}$ 产生氧气, BGM5 和 BGM9 支架在 $2 \mathrm{mmol} / \mathrm{L} \mathrm{H}_{2} \mathrm{O}_{2}$ 溶液中持续 产生氧气, 饱和氧浓度分别达到 8.4 和 $11 \mathrm{mg} / \mathrm{L}$ 。细 胞实验结果表明 BGM 支架对细胞增殖和成骨分化 具有促进作用。因此, BGM 支架具有消除骨缺损处 过量 $\mathrm{H}_{2} \mathrm{O}_{2}$ 并缓解缺氧状态的功能, 在骨缺损修复 领域具有较大的应用潜力。

\section{参考文献:}

[1] SHIEKH P A, SINGH A, KUMAR A. Oxygen releasing antioxidant cryogel scaffolds with sustained oxygen delivery for tissue engineering applications. ACS Applied Materials \& Interfaces, 2018, 10(22): 18458-18469.

[2] LOVETT M, LEE K, EDWARDS A, et al. Vascularization strategies for tissue engineering. Tissue Engineering Part B: Reviews, 2009, 15(3): 353-370.

[3] BECQUART P, CAMBON-BINDER A, MONFOULET L E, et al. Ischemia is the prime but not the only cause of human multipotent stromal cell death in tissue-engineered constructs in vivo. Tissue Engineering Part A, 2012, 18(19/20): 2084-2094.

[4] PISU M, LAI N, CINCOTTI A, et al. Modeling of engineered cartilage growth in rotating bioreactors. Chemical Engineering Science, 2004, 59: 5035-5040.

[5] TOURI M, MOZTARZADEH F, OSMAN N A A, et al. 3D-printed biphasic calcium phosphate scaffolds coated with an oxygen generating system for enhancing engineered tissue survival. Material Science and Engineering C, 2018, 84: 236-242.

[6] LÜ X G, LI Z, CHEN S Y, et al. Structural and functional evaluation of oxygenating keratin/silk fibroin scaffold and initial assessment of their potential for urethral tissue engineering. Biomaterials, 2016, 84: 99-110.

[7] LEE E M, JUNG J I, ALAM Z, et al. Effect of an oxygengenerating scaffold on the viability and insulin secretion function of porcine neonatal pancreatic cell clusters. Xenotransplantation, 2018, 25(2): e12378.

[8] KUMAR S, ADJEI I M, BROWN S, et al. Manganese dioxide nanoparticles protect cartilage from inflammation-induced oxidative stress. Biomaterials, 2019, 224: 119467.

[9] BIZEAU J, TAPEINOS C, MARELLA C, et al. Synthesis and characterization of hyaluronic acid coated manganese dioxide microparticles that act as ROS scavengers. Colloids and Surfaces B: Biointerfaces, 2017, 159: 30-38.

[10] LI Q, REN J J, CHEN Q B, et al. A HMCuS@ $\mathrm{MnO}_{2}$ nanocomplex responsive to multiple tumor environmental clues for photoacoustic/ fluorescence/magnetic resonance trimodal imaging-guided and enhanced photothermal/photodynamic therapy. Nanoscale, 2020, 12(23): 12508-12521.

[11] WANG Y D, SONG S Z, LU T, et al. Oxygen-supplementing mesoporous polydopamine nanosponge with $\mathrm{WS}_{2}$ QDs-embedded for CT/MSOT/MR imaging and thermoradiotherapy of hypoxic cancer. Biomaterials, 2019, 220: 119405.

[12] ANAND A, LALZAWMLIANA V, KUMAR V, et al. Preparation and in vivo biocompatibility studies of different mesoporous bioactive glasses. Journal of the Mechanical Behavior of Biomedical Materials, 2019, 89: 89-98.

[13] KARGOZAR S, MONTAZERIAN M, HAMZEHLOU S, et al. Mesoporous bioactive glasses: promising platforms for antibacterial strategies. Acta Biomaterialia, 2018, 81: 1-19.

[14] SAEID K, FRANCESCO B, SEPIDEH H, et al. Bioactive glasses entering the mainstream. Drug Discovery Today, 2018, 23: S1359644618300394.

[15] MUBINA M S K, SHAILAJHA S, SANKARANARAYANAN R, et al. In vitro bioactivity, mechanical behavior and antibacterial properties of mesoporous $\mathrm{SiO}_{2}-\mathrm{CaO}-\mathrm{Na}_{2} \mathrm{O}-\mathrm{P}_{2} \mathrm{O}_{5}$ nano bioactive glass ceramics. Journal of the Mechanical Behavior of Biomedical Materials, 2019, 100: 103379.

[16] FU S Y, ZHU M, ZHU Y F. Organosilicon polymer-derived ceramics: an overview. Journal of Advanced Ceramics, 2019, 8(4): 457-478.

[17] CHEN Z, SUN X H, SHANG Y P, et al. Dense ceramics with complex shape fabricated by 3D printing: a review. Journal of Advanced Ceramics, 2021, 10(2): 195-218.

[18] YANG L L, ZENG X J, DITTA A, et al. Preliminary 3D printing of large inclined-shaped alumina ceramic parts by direct ink writing. Journal of Advanced Ceramics, 2020, 9(3): 312-319.

[19] ZHAO Z, ZHOU G X, YANG Z H, et al. Direct ink writing of continuous $\mathrm{SiO}_{2}$ fiber reinforced wave-transparent ceramics. Journal of Advanced Ceramics, 2020, 9(4): 403-412.

[20] WU C T, LUO Y X, CUNIBERTI G, et al. Three-dimensional printing of hierarchical and tough mesoporous bioactive glass scaffolds with a controllable pore architecture, excellent mechanical strength and mineralization ability. Acta Biomaterialia, 2011, 7(6): 2644-2650.

[21] LI L, HU H X, ZHU Y F, et al. 3D-printed ternary $\mathrm{SiO}_{2}-\mathrm{CaO}-\mathrm{P}_{2} \mathrm{O}_{5}$ bioglass-ceramic scaffolds with tunable compositions and properties for bone regeneration. Ceramics International, 2019, 45(8): 10997-11005. 
[22] YE X, JIANG X, CHEN L, et al. Effect of manganese dioxide crystal structure on adsorption of $\mathrm{SO}_{2}$ by DFT and experimental study. Applied Surface Science, 2020, 521: 146477.

[23] REN L L, ZHOU D Y, WANG J J, et al. Biomaterial-based flower-like $\mathrm{MnO}_{2} @$ carbon microspheres for rapid adsorption of amoxicillin from wastewater. Journal of Molecular Liquids, 2020, 309: 113074.

[24] CHEN Y, YE D L, WU M Y, et al. Break-up of two-dimensional $\mathrm{MnO}_{2}$ nanosheets promotes ultrasensitive $\mathrm{pH}$-triggered theranostics of cancer. Advanced Materials, 2014, 26(41): 7019-7026.

[25] HU X N, SHI L Y, ZHANG D S, et al. Accelerating the decomposition of $\mathrm{KMnO}_{4}$ by photolysis and auto-catalysis: a green approach to synthesize a layered birnessite-type $\mathrm{MnO}_{2}$ assembled hierarchical nanostructure. RSC Advances, 2016, 6(17): 14192-14198.

[26] SHAABANI A, AFARIDOUN H, SHAABANI S. Natural hydroxyapatite-supported $\mathrm{MnO}_{2}$ : a green heterogeneous catalyst for selective aerobic oxidation of alkylarenes and alcohols. Applied Organometallic Chemistry, 2016, 30(9): 772-776.

[27] HAO X L, ZHAO J Z, SONG Y H, et al. Surfactant-assisted synthesis of birnessite-type $\mathrm{MnO}_{2}$ nanoflowers. Journal of Nano Research, 2018, 53: 1-6.

[28] JING X N, XU Y Z, LIU D M, et al. Intelligent nanoflowers: a full tumor microenvironment-responsive multimodal cancer theranostic nanoplatform. Nanoscale, 2019, 11(33): 15508-15518.

[29] HE D G, HE X X, WANG K, et al. Redox-responsive degradable honeycomb manganese oxide nanostructures as effective nanocarriers for intracellular glutathione-triggered drug release. Chemical Communications, 2014, 51(4): 776-779.

[30] CHEN B, WANG F. $\mathrm{NaYbF}_{4} @ \mathrm{CaF}_{2}$ core-satellite upconversion nanoparticles: one-pot synthesis and sensitive detection of glutathione. Nanoscale, 2018, 10(42): 19898-19905.

[31] WANG Z Z, ZHANG Y, JU E G, et al. Biomimetic nanoflowers by self-assembly of nanozymes to induce intracellular oxidative damage against hypoxic tumors. Nature Communications, 2018, 9(1): 3334

[32] YAN X X, YU C Z, ZHOU X F, et al. Highly ordered mesoporous bioactive glasses with superior in vitro bone-forming bioactivities. Angewandte Chemie International Edition, 2004, 116: 6106-6110.

[33] DENG R R, XIE X J, VENDRELL M, et al. Intracellular glutathione detection using $\mathrm{MnO}_{2}$-nanosheet-modified upconversion nanoparticles. Journal of the American Chemical Society, 2011, 133(50): 20168-20171.

[34] ZHANG L H, LIAN J S, WU L Y, et al. Synthesis of a thin-layer $\mathrm{MnO}_{2}$ nanosheet-coated $\mathrm{Fe}_{3} \mathrm{O}_{4}$ nanocomposite as a magnetically separable photocatalyst. Langmuir, 2014, 30(23): 7006-7013.
[35] FRÖHLICH M, GRAYSON W L, WAN L Q, et al. Tissue engineered bone grafts: biological requirements, tissue culture and clinical relevance. Current Stem Cell Research \& Therapy, 2008, 3(4): 254-264.

[36] AZIZI F, HEIDARI F, FAHIMIPOUR F, et al. Evaluation of mechanical and biocompatibility properties of hydroxyapatite/manganese dioxide nanocomposite scaffolds for bone tissue engineering application. International Journal of Applied Ceramic Technology, 2020, 17(5): 2439-2449.

[37] HSIEH T E, LIN S J, CHEN L C, et al. Optimizing an injectable composite oxygen-generating system for relieving tissue hypoxia. Frontiers in Bioengineering and Biotechnology, 2020, 8: 511.

[38] LACY F, KAILASAM M T, O'CONNOR D T, et al. Plasma hydrogen peroxide production in human essential hypertension role of heredity, gender, and ethnicity. Hypertension, 2000, 36(5): 878-884.

[39] BURGOYNE J R, OKA S I, ALE-AGHA N, et al. Hydrogen peroxide sensing and signaling by protein kinases in the cardiovascular system. Antioxidants \& Redox Signaling, 2013, 18(9): 1042-1052.

[40] WANG J Q, ZHANG Y Q, ARCHIBONG E, et al. Leveraging $\mathrm{H}_{2} \mathrm{O}_{2}$ levels for biomedical applications. Advanced Biosystems, 2017, 1(9): 1700084.

[41] TAPEINOS C, LARRAÑAGA A, SARASUA J R, et al. Functionalised collagen spheres reduce $\mathrm{H}_{2} \mathrm{O}_{2}$ mediated apoptosis by scavenging overexpressed ROS. Nanomedicine, 2018, 14(7): 2397-2405.

[42] JÄGER E, HÖCHERL A, JANOUŠKOVÁ O, et al. Fluorescent boronate-based polymer nanoparticles with reactive oxygen species (ROS)-triggered cargo release for drug-delivery applications. Nanoscale, 2016, 8(13): 6958-6963.

[43] DEEPAGAN V G, KWON S, YOU D G, et al. In situ diselenidecrosslinked polymeric micelles for ROS-mediated anticancer drug delivery. Biomaterials, 2016, 103: 56-66.

[44] WU C T, RAMASWAMY Y, ZHU Y F, et al. The effect of mesoporous bioactive glass on the physiochemical, biological and drug-release properties of poly(DL-lactide-co-glycolide) films. Biomaterials, 2009, 30(12): 2199-2208.

[45] WU C T, CHANG J. Degradation, bioactivity, and cytocompatibility of diopside, akermanite, and bredigite ceramics. Journal of Biomedical Materials Research Part B Applied Biomaterials, 2010, 83B(1): 153-160.

[46] NAWAZ Q, REHMAN M A U, ROETHER J A, et al. Bioactive glass based scaffolds incorporating gelatin/manganese doped mesoporous bioactive glass nanoparticle coating. Ceramics International, 2019, 45(12): 14608-14613. 